\title{
Significance of microsatellite instability (MSI) for colorectal cancer following adjuvant therapy with doxifluridine
}

\author{
Toshiaki Watanabe $\cdot$ Keiji Matsuda $\cdot$ Soichiro Ishihara \\ Keijiro Nozawa $\cdot$ Tamuro Hayama $\cdot$ Hideki Yamada • \\ Hisae Iinuma
}

Received: 15 December 2010/Accepted: 22 December 2010/Published online: 15 January 2011

(C) Springer Science+Business Media, LLC 2011

\section{Letter to Editor}

We read with interest the study by Kang and colleagues published in the recent issue of Medical Oncology [1]. They examined the usefulness of mcrosatellite instability (MSI) status as a predictive factor for stage II or III colorectal cancer (CRC) patients who received adjuvant doxifluridine therapy. MSI-H showed a favorable survival trend for overall survival and significant survival benefit for relapse-free survival. In a univariate analysis, the doxifluridine-treated patients with MSI-H showed improved relapse-free survival compared to those with low or stable MSI (MSI-L/S), however, in a multivariate analysis, MSI-H was not significantly associated with relapse-free survival. Therefore, they concluded that MSI-H patients with stage II or III CRC did not seem to benefit from doxifluridine adjuvant therapy. As the author stated, the predictive role of the MSI status remains unclear at the present time. We have previously demonstrated that adjuvant chemotherapy is effective for stage II and stage MSI CRC [2]. However, other studies show conflicting results [3]. Kang et al. showed results of patients who received doxifluridine adjuvant therapy and there have been very few studies that have addressed the effect of doxifluridine in MSI CRC in adjuvant setting. In this sense, Kang's study presented an important result. However, to draw their conclusion there is a major issue that needs to be discussed.

Kang et al. examined 564 stage II or III patients, however, more than half of the patients were stage II (290 patients). Although, effectiveness of adjuvant chemotherapy has been well established in stage III CRC, its role in stage II CRC is

T. Watanabe $(\bowtie) \cdot$ K. Matsuda $\cdot$ S. Ishihara $\cdot$ K. Nozawa

T. Hayama $\cdot$ H. Yamada $\cdot$ H. Iinuma

Department of Surgery, Teikyo University School of Medicine,

2-11-1 Kaga, Itabashi-ku, Tokyo 173-8605, Japan

e-mail: toshwatanabe@yahoo.co.jp still unclear. Furthermore, in our previous study, we selected high-risk stage II patients, because these patients may be good candidates for adjuvant therapy. Also, it should be noted that in Kang's study there were only 13 MSI stage III patients and 30 MSI stage II patients. These numbers seem too small in order to evaluate any effect of adjuvant therapy. In fact, overall survival curves and relapse-free survival curves in both all patients and patients who received adjuvant therapy all show better outcome in MSI patients than in MSI-L/S patients. To accurately evaluate the efficacy of doxifluridine adjuvant therapy, larger numbers of high-risk stage II and stage III patients are necessary.

Until above issue is sufficiently addressed, we believe it is premature to conclude that MSI-H patients with stage II or III CRC did not benefit from doxifluridine adjuvant therapy.

Conflict of interest The authors have no conflict of interest to declare.

\section{References}

1. Kang BW, Kim JG, Lee SJ, Chae YS, Moon JH, Sohn SK, et al. Clinical significance of microsatellite instability for stage II or III colorectal cancer following adjuvant therapy with doxifluridine. Med Oncol. 16 Oct 2010 [Epub ahead of print].

2. Watanabe T, Wu TT, Catalano PJ, Ueki T, Satriano R, Haller DG, et al. Molecular predictors of survival after adjuvant chemotherapy for colon cancer. N Engl J Med. 2001;344:1196-206.

3. Ribic CM, Sargent DJ, Moore MJ, Thibodeau SN, French AJ, Goldberg RM, et al. Tumor microsatellite-instability status as a predictor of benefit from fluorouracil-based adjuvant chemotherapy for colon cancer. N Engl J Med. 2003;349:247-57. 